This item was submitted to Loughborough's Research Repository by the author.

Items in Figshare are protected by copyright, with all rights reserved, unless otherwise indicated.

\title{
Rich interaction and feedback supported mammographic training: A trial of an augmented reality approach
}

PLEASE CITE THE PUBLISHED VERSION

https://doi.org/10.1007/978-3-319-60964-5_33

\section{PUBLISHER}

(C) Springer

VERSION

AM (Accepted Manuscript)

\section{PUBLISHER STATEMENT}

This work is made available according to the conditions of the Creative Commons Attribution-NonCommercialNoDerivatives 4.0 International (CC BY-NC-ND 4.0) licence. Full details of this licence are available at: https://creativecommons.org/licenses/by-nc-nd/4.0/

\section{LICENCE}

CC BY-NC-ND 4.0

\section{REPOSITORY RECORD}

Tang, Qiang, Yan Chen, and Alastair G. Gale. 2019. "Rich Interaction and Feedback Supported Mammographic Training: A Trial of an Augmented Reality Approach”. figshare. https://hdl.handle.net/2134/32519. 


\title{
Rich interaction and feedback supported mammographic training: a trial of an Augmented Reality approach
}

\author{
Qiang Tang ${ }^{1}$, Yan Chen ${ }^{1}$, and Alastair G Gale ${ }^{1}$ \\ ${ }^{1}$ Applied Vision Research Centre, Loughborough University, Loughborough, UK \\ Y.Chen@lboro.ac.uk
}

\begin{abstract}
The conventional 'keyboard and workstation' approach allows complex medical image presentation and manipulation during mammographic interpretation. Nevertheless, providing rich interaction and feedback in real time for navigational training or computer assisted detection of disease remains a challenge. Through computer vision and state of the art AR (Augmented Reality) technique, this study proposes an 'AR mammographic workstation' approach which could support workstationindependent rich interaction and real-time feedback. This flexible AR approach explores the feasibility of facilitating various mammographic training scenes via $\mathrm{AR}$ as well as its limitations.
\end{abstract}

Keywords: Augmented Reality · Mammographic Training · Interaction - Feedback

\section{Introduction}

To maintain quality in the UK Breast Screening Programme, a nationwide mandatory self-assessment scheme has been developed and deployed across the UK for the past 30 years. The current work presented here builds on this and addresses the particular need for supplying richer interaction and real time feedback in medical training or assessment by incorporating AR based interaction and feedback.

Training plays a significant role in maintaining mammographic interpretation skills as demonstrated by the UK self-assessment scheme (Personal Performance in Mammographic Screening PERFORMS ${ }^{\circledR}$ ) [1]. According to [2], effective and sufficient training keeps being challenged by the shortage of high cost DICOM mammographic workstations. Early research [3] and [4] suggests appropriate interaction approaches could highly facilitate mammographic training. [6] and [7] indicate that feedback, as a critical training characteristic, provides a positive influence on trainee behaviour in medical training. The fast development of AR devices opens new opportunities to incorporating rich interaction and feedback with medical training without considering time or location [8]. Also, [5] suggests appropriate novel interaction approaches are important components of employing various display size in different mammographic interpretation training situations. The current study describes a device-independent AR approach 
which can provide interaction and feedback to support medical interpretation training in real time.

\section{Method}

The initial AR approach is conceptually constructed as the following steps:

1) A series of mammographic cases which are either normal or have malignant or benign radiologic features are first examined by an expert radiologist on a workstation who annotates the cases. If any malignant or benign features are present on a case, then there are also feedback marks (areas of interest identified by radiologists) associated with the case which can be presented to the trainee radiologists by the AR system.

2) A trainee radiologist wears the AR system as they inspect the mammographic images. Once the AR system appropriately identifies the particular case being examined then appropriate feedback information can be displayed on the AR system. The AR system has a forward facing camera and a display which enables the scene (i.e. here the mammographic images) to be captured and feedback to be overlaid on the viewed scene.

3) Computer vision (object recognition) is implemented for this AR approach to effectively detect the working overall scene in front of the radiologist and extract the actual screen areas of the workstation into the AR system as time-sequenced images. These time-sequenced images are captured in real time so that a perspective transformation has to be applied to enable accurate overlaying feedback marks for each image. This approach then allows for the radiologist to move their head whilst constantly permitting accurate overlay of feedback information on the viewed mammographic cases. The perspective transformation [11] is a general $3 * 3$ matrix expression for spatial transformations. Then an inverse transformation of the above enables pathology feedback information to overlay the real world scene appropriately.

4) As a result, feedback marks are co-registered with the transformed images and presented to the radiologist via the AR display. This feedback information ia only visible to the radiologist wearing the AR device.

The initial setup (Figure 1) comprises a GE mammographic workstation, Google Glass and an Android virtual machine which is used for image processing. Considering that Google Glass has a critical performance limitation of image processing capability, a subsequent setup (Figure 2) employs a simulated AR approach which uses a fixed camera (numbered as 3) and a laptop (numbered as 2) instead of Google Glass and the Android virtual machine to appropriately simulate an AR environment. Both setups are unobtrusive and allow radiologists quickly to access response feedback information. The only difference is that augmented views can be presented to the user via the laptop screen rather than directly presented by the see-through display of a wearable AR, whereas the laptop-based AR approach maximally provides a simulated AR training scene. 
A further OCR approach recognises radiological case identity. Additionally, a natural writing method is implemented which allows a recognised stylus (numbered as 1 in Figure 2) held by the radiologist logging its position on the workstation images. These both allow rich interaction and synchronising of the cases on the workstation and the AR device so that the same mammographic case displayed on the dedicated workstation is loaded from the PERFORMS ${ }^{\circledR}$ database and is also presented on the AR device at the same time.

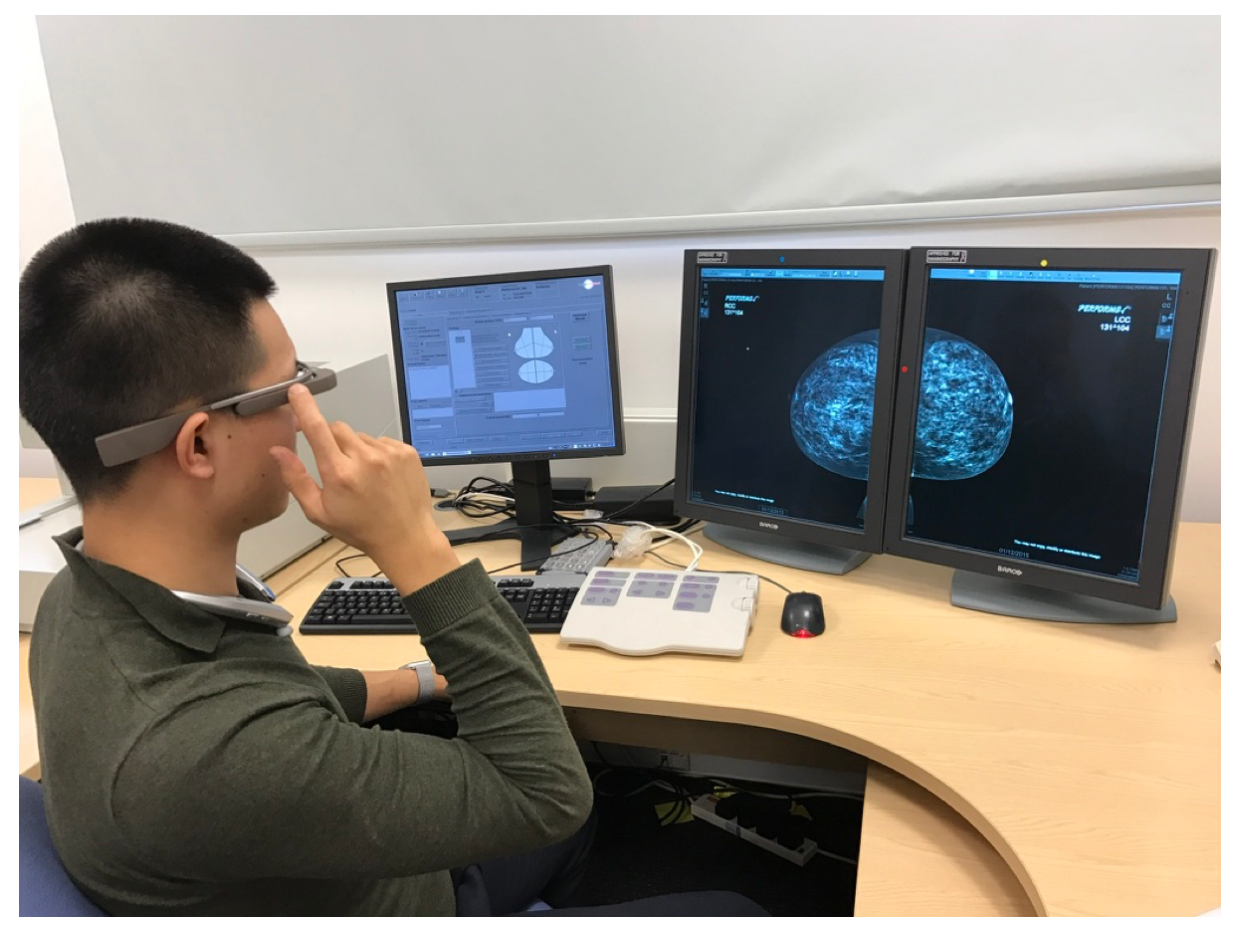

Fig. 1. The setup of the Google Glass AR environment

An effectively designed mammographic training process is able to incorporate virtual objects with human vision based reality so that augmented vision is obtained. Simultaneously, it allows seamless interaction between the human, the workstation and the AR device so that behaviour-dependent feedback information is supplied in real time. Such an augmented interaction is achieved via computer vision. Figure 3 shows the overall concept of incorporating augmented interaction and vision with conventional mammographic training.

An experimental AR prototype is implemented to support the delivery of mammographic training. The detailed workflow in Figure 4 shows the mechanism of incorporating AR with conventional mammographic training. In this 


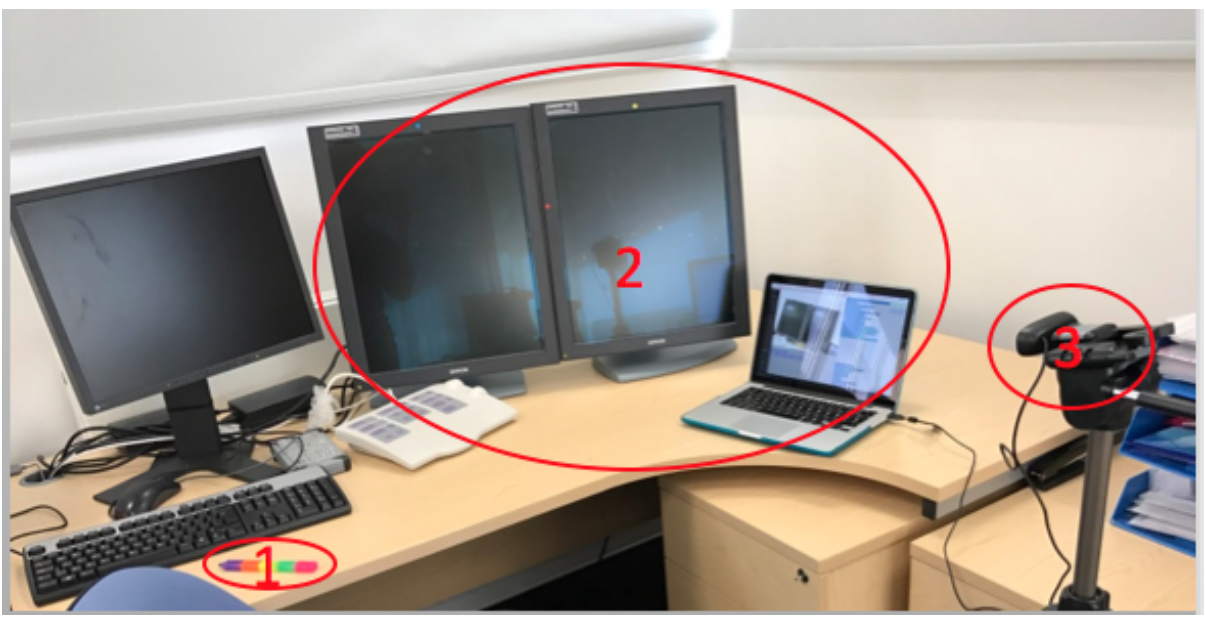

Fig. 2. The setup of the laptop-based AR environment has three essential elements: a GE workstation, a single camera and a laptop.

Figure, blue labels represent the general workflow of existing training approaches. Labels E and F respectively represent existing approaches and the AR approach. The AR approach workflow consists of augmented vision sub-workflow and augmented interaction sub-workflow, which are represented by green and grey labels individually. Augmented vision and augmented interaction are described separately below in numbered steps.

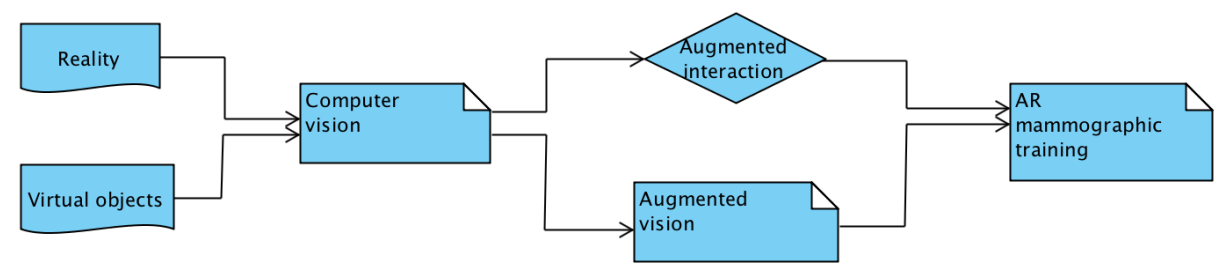

Fig. 3. The concept of AR mammographic training.

i) Augmented vision (green labels in Figure 4)

a) (1 in Figure 4) The radiologist's view is time-sequentially captured by a high resolution AR camera. These time-sequenced images comprise the training scene (A in Figure 4). 


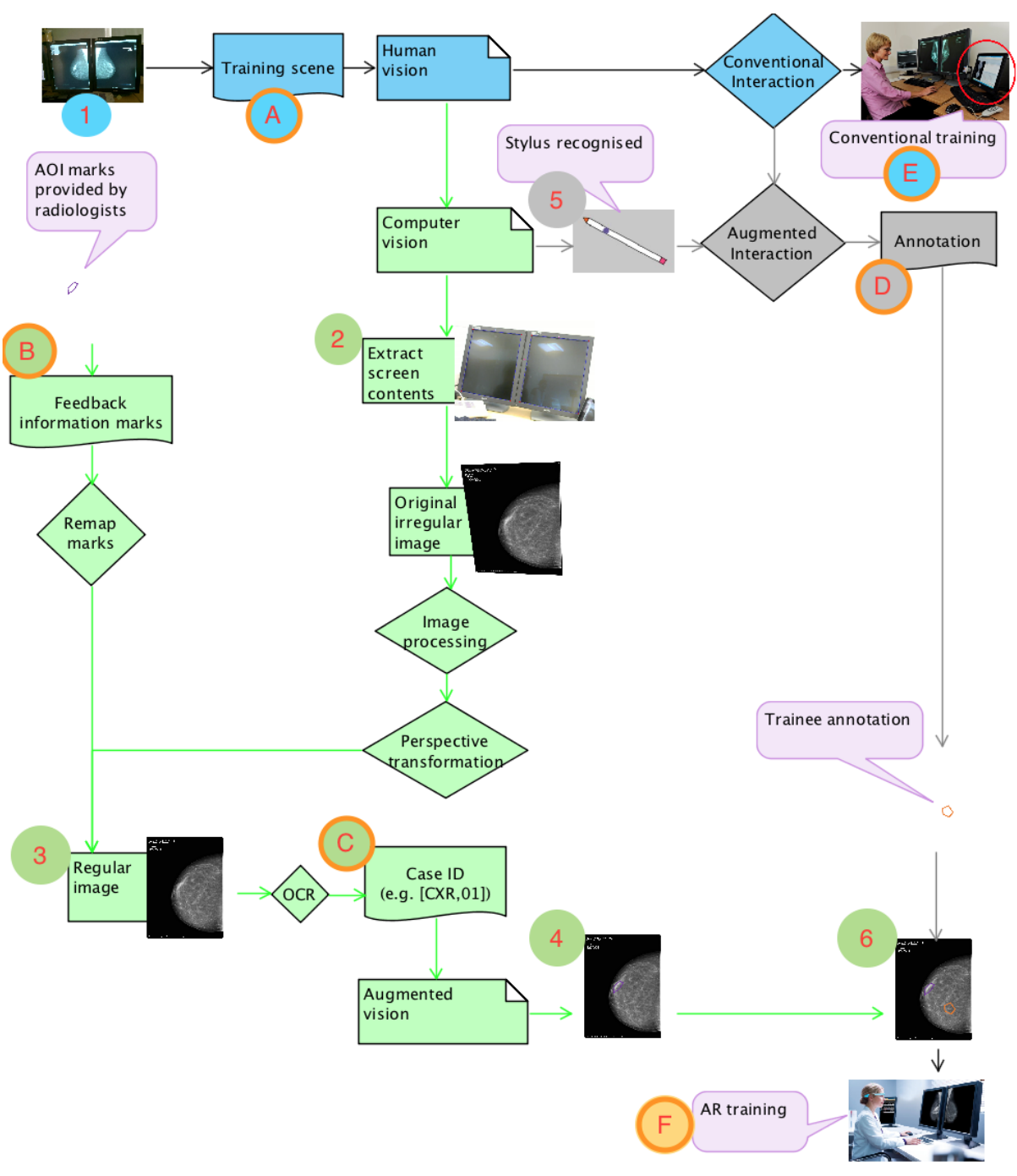

Fig. 4. The workflow of AR mammographic training. 
b) (2 in Figure 4) Through computer vision, the AR prototype extracts the dedicated mammographic workstation screens and their contents from the overall scene.

c) (3 in Figure 4) Through performing further image processing, including hough transformation [10] and perspective transformation [11], the irregular appearing mammographic image captured by the single camera is then reconstructed as a corrected rectangular medical image. To be adapted for dedicated workstations or standard lightweight devices, screen distribution information is calculated after the hough transformation so that the AR approach can support complex training scenes with more than two devices which are different in their display size.

d) (4 in Figure 4) Feedback information marks are provided by radiologists (B in Figure 4) and case identity is recognised via computer vision (C in Figure 4) are both synchronised with the reconstructed regular mammographic image.

ii) Augmented interaction (grey labels in Figure 4)

a) (5 in Figure 4) The prototype is designed to recognise a stylus and track the natural handwriting behaviour of the radiologist via the stylus. This allows virtual annotation feedback (D in Figure 4) to be generated and incorporated with the original mammographic images.

Finally, the enhanced mammographic image overlaying the expert radiologist feedback information and trainee annotations is constructed by assembling the training scene (A in Figure 4), the feedback information marks (B in Figure $4)$, the recognised mammographic case identity ( $\mathrm{C}$ in Figure 4$)$ and the trainee specified annotations (D in Figure 4). A sequential inverse perspective transformation of 3 in Figure 4 is applied to accurately project the overlaid virtual information onto the real training scene. Therefore, AR training ( $\mathrm{F}$ in Figure 4) can be independent of any dedicated DICOM viewing workstation or software and enables superimposition of rich feedback information and interaction in real time via assembling reality and accurate virtual information registration as needed. Compared to conventional training ( $\mathrm{E}$ in Figure 4) which requires transcribing on another device [9], the AR approach can be vendor neutral and adapted for various viewing platforms (e.g. dedicated workstation or handheld device).

\section{Results}

The proposal demonstrates that $\mathrm{AR}$ can deliver rich interaction interpretation training via dynamically remapping radiological feedback into the real world using perspective transformation. A natural writing method (a stylus recognised through computer vision) enables rich interaction and feedback as needed. Feedback presentation and interaction both suggest a pixel level experimental accuracy for hardware. Synchronising of mammographic cases, both on a workstation and an AR system, is enabled, although complicated image manipulations are 
not yet encompassed. The AR approach is device-independent so that it can be used for different training scenarios (for example, a mixted environment of a tablet and a conventional workstation). This flexible generic approach works without any artificial marker for object registration and no calibration is required.

The registration accuracy of the overlaid feedback marks is gauged to be at a pixel level after correct detection of the training scene and workstation monitor screens via computer vision. Two metrics of hardware, camera resolution and display resolution, limit the accuracy of AR registration.

Hand input via the stylus is estimated by a series of carefully designed visionguided experiments. Visual cues are used to identify the positions of the stylus components which are distinguished by different colours (Figure 5: the drawing path of the stylus is represented by pink colour and a coloured dot indicates the centre of the stylus). To establish accuracy the AR prototype generates random points in the human visual field and requires a participant to identify each point by the stylus. The distance for a computer generated point to each participant identifying point is then calculated automatically and the mean distance derived. Repeated experiments show that the hand input accuracy (mean distance between computer generated points and identified points) is within \pm 5 pixels. Nevertheless, hand input is significantly affected by handheld stability and the accuracy of the stylus detection via computer vision. High resolution and large size images are also factors in improving hand input accuracy. [12] claim consistent results while transcribing mammographic image feature positions.

\section{Limitation}

Conventional complicated medial image manipulations, for instance, zooming or panning are not currently mirrored in the proposed AR approach. Due to the image processing requirements and complex calculation procedures high performance hardware is essential for AR. Appropriate parallel computing for computer vision by GPU programming could significantly accelerate the process of AR detection and registration. However, the scarcity of high performance AR devices limits the wide adoption of AR in radiology training. A compromised solution is to employ an individual computing unit (e.g. a high performance laptop) for the complex image processing while presenting augmented views to radiologists via a see-through display.

\section{Discussion}

Following the perceived need of situated medical education, particular complicated medical training regarding difficulties in responding and gaining quick feedback information associated with self performance [13], immersive and high realistic situated training show the immense potential of enabling complicated perception of the training process and direct interaction by superimposing appropriate virtual information. Especially for mammographic training, there is a 
critical limitation in synchronising supplementary radiological case-associated information and allowing direction interaction or complex feedback. An important reason is the commercial edges maintained either by hardware or software vendors so that key technical details are not divulged to allow flexible training. AR provides a vendor neutral method which can superimpose device-independent interaction and feedback as needed via computer vision. It allows mammographic training to be independent of time, location, and device type because each realistic object is wrapped and complemented with a virtual property. For instance, a dedicated mammographic workstation and a tablet can form a flexible training scene together (Figure 5). However, input accuracy can be affected by display size and resolution.

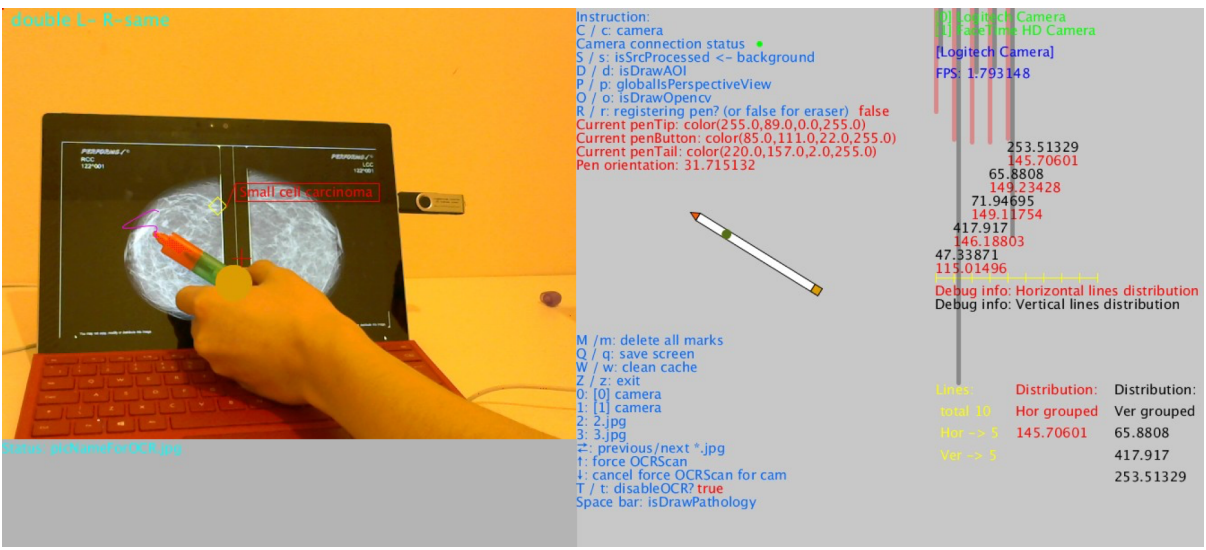

Fig. 5. AR Mammographic training with a tablet.

It is also acknowledged that existing wearable AR devices have no capability for high performance computing which is essentially required by image processing, in particular with breast screening. Incorporating an individual computing unit (e.g. a laptop with a dedicated graphic card) with a low cost AR display can be a superior compromise.

Although this study is concerned with mammographic images, the application is not only limited to mammographic screening training. Other similar training scenarios could also adopt this method to facilitate interaction and provide rich feedback. Moreover, incorporating contextual and accurate location-related CAD prompts can be achieved in interpretation training by recognising virtual contents (mammographic images) through computer vision.

In the future, appropriate experiments will be conducted to decide whether the AR training approach could effectively facilitate medical training quality by comparing AR and existing training approaches. 


\section{Conclusion}

It is concluded that it is feasible to employ Augmented Reality to deliver situated learning and facilitate perception of complex situations in mammographic training. AR is a superior interface which seamlessly connects reality with rich virtual interaction and overlaid vision. However, the current development platform could not realise the AR approach's full potential. Appropriate hardware performance is the critical important factor in the wide adoption of AR in medical imaging education. The AR approach described here provides a compromised solution to build a maximally simulated AR training environment. A future series of experiments will investigate possible mammographic image manipulations via $\mathrm{AR}$.

\section{References}

1. Chen, Y., Gale, A., Scott, H.: Mammographic interpretation training: what exactly do film-readers want? Breast Cancer Research, 10(Suppl 3), p.P85 (2008). doi:10. $1186 /$ bcr 2083

2. Chen, Y., Gale, A.: Intelligent Computing Applications Based on Eye Gaze: Their Role in Medical Image Interpretation. International Conference on Intelligent Computing, 93(Chapter 43), pp.320325 (2010). doi:10.1007/978-3-642-14831-6_43

3. Preim, B.: HCI in Medical Visualization. Dagstuhl Follow-Ups, 2, p.310 (2011). doi:10.4230/DFU.Vol2.SciViz.2011.292

4. Weiss, D.L., Siddiqui, K.M., Scopelliti, J.: Radiologist assessment of PACS user interface devices. Journal of the American College of Radiology : JACR, 3(4), pp.265273 (2006). doi:10.1016/j.jacr.2005.10.016

5. Chen, Y., Gale, A.G., Scott, H.: Mammographic interpretation training in the UK: current difficulties and future outlook. In B. Sahiner \& D. J. Manning, eds. SPIE Medical Imaging. SPIE, pp. 72631C12 (2009). doi:10.1117/12.810730

6. Wood, B.P.: Feedback: A Key Feature of Medical Training. Radiology, 215(1), pp.1719 (2000). doi:10.1148/radiology.215.1.r00ap5917

7. Karl, K.A., O'Leary-Kelly, A.M., Martocchio, J.J.: The Impact of Feedback and Self-Efficacy on Performance in Training. Journal of Organizational Behavior, 14(4), pp.379394 (1993). doi:10.2307/2488290?ref=search-gateway: c3129bc069220854d7dbb24db580bf8f

8. Chen, Y., Gale, A., Scott, H.: Anytime, anywhere mammographic interpretation training. Contemporary Ergonomics, pp.375380 (2008). doi:10.1201/9780203883259. ch59

9. Gale, A.G. PERFORMS: a self-assessment scheme for radiologists in breast screening. Seminars in Breast Disease, 6(3), pp.148152 (2003). doi:10.1053/j.sembd.2004. 03.006

10. Mukhopadhyay, P., Chaudhuri, B.B. A survey of Hough Transform. Pattern Recognition (), 48(3), pp.9931010 (2015). doi:10.1016/j.patcog.2014.08.027

11. Wolberg, G. Geometric Transformation Techniques for Digital Images: a Survey, Department of Computer Science, Columbia University. (1988).

12. Hatton, J.W. et al. Accuracy of transcribing locations on mammograms: implications for the user interface of a system to record and assess breast screening decisions. In D. P. Chakraborty \& E. A. Krupinski, eds. Proceedings of the SPIE. SPIE, pp. 3241 (2003). doi:10.1117/12.480086 
13. Kamphuis, C. et al. Augmented reality in medical education? Perspectives on Medical Education, 3(4), pp.300311 (2014). doi:10.1007/s40037-013-0107-7 